\title{
Tanshinone IIA improves diabetes mellitus via the NF-kB-induced AMPK signal pathway
}

\author{
FENG-YI YUAN ${ }^{1 *}$, MIN ZHANG $^{2 *}$, PING XU $^{1}$, DAN XU $^{1}$, PING CHEN $^{2}$, \\ MIN REN ${ }^{2}$, QIN SUN ${ }^{2}$, JING-YAN CHEN ${ }^{2}$, JUAN DU $^{2}$ and XIA-LIAN TANG ${ }^{2}$ \\ ${ }^{1}$ Department of Endocrinology and Metabolism, Second Affiliated Hospital of Jinan University, Shenzhen, \\ Guangdong 518020; ${ }^{2}$ Geriatric Department of Endocrinology, Sichuan Province People's Hospital \\ and Sichuan Academy of Medical Sciences, Chengdu, Sichuan 610072, P.R. China
}

Received August 18, 2017; Accepted January 3, 2018

DOI: $10.3892 /$ etm.2018.6674

\begin{abstract}
Diabetes mellitus (DM) is a systemic metabolic disease. Tanshinone IIA (Tan-IIA) presents potential benefits for DM. The purpose of this study was to investigate the efficacy of Tan-IIA in type 2 DM rats and explore its potential mechanism in renal cells. A type $2 \mathrm{DM}$ rat model was established and administered with Tan-IIA or PBS. It was demonstrated that Tan-IIA treatment significantly reduced levels of total cholesterol, non-esterified fatty acids, total triglyceride and low-density lipoprotein cholesterol in experimental DM rats compared with the control group. The results indicated that Tan-IIA treatment significantly decreased levels of interleukin (IL)-8, tumor necrosis factor- $\alpha$, C-reactive protein and IL-6. It was identified that Tan-IIA treatment significantly decreased nuclear factor- $\kappa$ B levels and significantly elevated 5 ' adenosine monophosphate-activated protein kinase levels. Western blot analysis indicated that Tan-IIA elevated immunocyte precipitation in renal cells. Furthermore, Tan-IIA treatment improved lipid metabolism, glucose metabolism, insulin resistance and body weight of type 2 DM rats. In conclusion, Tan-IIA administration may inhibit inflammatory cytokines and alleviate type 2 DM symptoms in experimental rats.
\end{abstract}

\section{Introduction}

Diabetes mellitus (DM) is a metabolic disease that is characterized by insulin secretion defects $(1,2)$. DM is also an

Correspondence to: Professor Min Zhang, Geriatric Department of Endocrinology, Sichuan Province People's Hospital and Sichuan Academy of Medical Sciences, $32 \mathrm{~W}$ 2nd Section, 1st Ring Road (West), Chengdu, Sichuan 610072, P.R. China

E-mail: zhangmindoctor@outlook.com

*Contributed equally

Key words: Tanshinone IIA, type 2 diabetes mellitus, inflammation, nuclear factor- $\kappa \mathrm{B}, 5^{\prime}$ adenosine monophosphate-activated protein kinase heterogeneous disease, which can be classified into type 1 and type 2 DM (3). Diabetic cardiomyopathy is often caused by metabolic disorders and microvascular lesions in patients with DM, and diabetic cardiomyopathy is responsible for inflammation and apoptosis of myocardial tissue (2-4). Diabetic cardiomyopathy is a major risk factor for the development of insulin resistance in patients with DM (5). The occurrence of hyperglycemia and metabolic syndrome may lead to the initiation and development of diabetic cardiomyopathy in patients with DM (6). These metabolic imbalances induced by diabetic cardiomyopathy may result in disturbed metabolism (7). Therefore, it is essential to understand the potential mechanism of DM to treat DM-induced metabolic syndrome in patients.

Tanshinone IIA (Tan-IIA) is a traditional Chinese medicine, which is extracted from Danshen (Salvia miltiorrhiza) and has been clinically used for the treatment of human cardiovascular and inflammatory diseases $(8,9)$. A previous study demonstrated that Tan-IIA can inhibit human cancer cell growth, and affects cell cycle regulation, cell proliferation, apoptosis and DNA synthesis (10). In addition, Tan-IIA significantly reduces lipopolysaccharide-induced acute lung injury by inhibiting inflammation and apoptosis in mice (11). Furthermore, renal fibrosis and inflammation can be attenuated by Tan-IIA via altering the expression of transforming growth factor- $\beta /$ Smad and nuclear factor (NF)- $\kappa \mathrm{B}$ signaling pathways in nephrectomized rats (12). Notably, Tan-IIA may serve a protective function against renal damage in type 2 DM through improving renal function, which could be a new evidence-based therapy for diabetic nephropathy (13).

In the present study, based on the link between inflammation and DM, it was assumed that Tan-IIA may have beneficial effects on type $2 \mathrm{DM}$ rats. The potential mechanisms mediated by Tan-IIA were investigated in a type $2 \mathrm{DM}$ rat model. To the best of our knowledge, this is the first study to demonstrate that Tan-IIA inhibits inflammation and alleviates type 2 DM symptoms through $\mathrm{NF}-\kappa \mathrm{B}$-induced 5 ' adenosine monophosphate-activated protein kinase (AMPK) signaling in experimental rats.

\section{Materials and methods}

Ethics statement. The protocols were approved by the Ethics Committee of Sichuan Province People's Hospital and Sichuan 
Academy of Medical Sciences (Chengdu, China). Anesthesia was administered using intravenous sodium pentobarbital (35 mg/kg, Sigma-Aldrich; Merck KGaA, Darmstadt, Germany).

Animal model. A total of 20 male Sprague-Dawley (SD) rats (age, 6-8 weeks; body weight, 320-340 g) were purchased from Charles River Laboratories (Beijing, China). All rats were housed in a temperature-controlled room $\left(25 \pm 1^{\circ} \mathrm{C}\right)$ with a 12 -h light/dark cycle. All rats were given accessed to food and water ad libitum. Type $2 \mathrm{DM}$ was induced in SD rats $(\mathrm{n}=20)$ using streptozotocin and high-fat diet, as described previously (14). The experimental rats with type $2 \mathrm{DM}$ were then divided into two groups ( $\mathrm{n}=10 \mathrm{in}$ each) and received Tan-IIA $(10 \mathrm{mg} / \mathrm{kg}$; Sigma-Aldrich; Merck KGaA) or PBS (10 mg/kg, $120 \mu \mathrm{l})$ by intragastric administration once every 2 days. Healthy male SD rats $(n=10$; age, 6-8 weeks; body weight, 320-340 g; Beijing University, Beijing, China) that did not receive treatment were used as controls and kept under the same conditions as the experimental rats. The treatments were continued for 8 weeks, and kidney weight $(\mathrm{KW})$ and body weight $(\mathrm{BW})$ were measured at the end of week 8. Total cholesterol (TC), non-esterified fatty acids (NEFAs), total triglyceride (TG) and total low density lipoprotein cholesterol (LDL-C) were measured at the end of week 8 as described previously (15).

Insulin tolerance tests. Experimental rats were fasted for $6 \mathrm{~h}$ after 8 weeks of treatment with Tan-IIA or PBS. Type 2 DM rats were injected with insulin (Sigma-Aldrich; Merck KGaA) intraperitoneally at $0.75 \mathrm{U} / \mathrm{kg}$ body weight. Blood glucose levels were measured $40 \mathrm{~min}$ after the insulin injection using a blood glucometer (Changsha Sannuo Biological Sensing Technology Co., Ltd., Changsha, China).

ELISA. Serum was isolated from $3 \mathrm{ml}$ peripheral blood using centrifugation at $4,000 \mathrm{x}$ g for $15 \mathrm{~min}$ at $4^{\circ} \mathrm{C}$. The concentrations of serum parameters were analyzed following treatment to identify mice in which chronic renal failure was induced by type 2 DM. ELISA kits were used to determine interleukin (IL)-6 (M6000B; R\&D Systems, Inc., Minneapolis, MN, USA), IL-10 (DY417; R\&D Systems, Inc.), C-reactive protein (CRP; MCRP00; R\&D Systems, Inc.), tumor necrosis factor (TNF)- $\alpha$ (MTA00B; R\&D Systems, Inc.), blood urea nitrogen (BUN; MBUN002; R\&D Systems, Inc.) and creatinine serum levels (KGR005; R\&D Systems, Inc.) in rats. The procedures were performed according to the manufacturer's protocols. The final results were recorded at $450 \mathrm{~nm}$ on an ELISA plate reader (Bio-Rad Laboratories, Inc., Hercules, CA, USA).

Western blot analysis. Rats were sacrificed and renal tissues were isolated from rats as described previously (16) for further analysis at the end of week 8. Renal cells were homogenized in $1 \mathrm{X}$ radioimmunoprecipitation assay buffer (Sigma-Aldrich; Merck KGaA) and western blotting was performed to analyze the protein expression. Briefly, protein concentrations were examined using a BCA protein assay (Invitrogen; Thermo Fisher Scientific, Inc., Waltham, MA, USA) and protein samples (40 $\mu \mathrm{g})$ were separated by $15 \%$ SDS-PAGE. Proteins were then blotted on a nitrocellulose membrane and blocked with $5 \%$ skimmed milk for $1 \mathrm{~h}$ at $37^{\circ} \mathrm{C}$. Membranes were incubated with primary antibodies against AMPK (1:2,000; cat. no. ab32047; Abcam, Cambridge, MA, USA), NF-кB p65 (1:2,000; cat. no. ab16502; Abcam), IL-6 (1:2,000; cat. no. ab9324; Abcam), IL-10 (1:2,000; cat. no. ab33471; Abcam), TNF- $\alpha$ (1:1,000; cat. no. ab6671; Abcam), CRP (1:2,000; cat. no. ab70010; Abcam), insulin receptor substrate (IRS)-1 (1:2,000; cat. no. ab52167; Abcam) and $\beta$-actin $\left(1: 2,000\right.$, cat. no. ab8226; Abcam) for $12 \mathrm{~h}$ at $4^{\circ} \mathrm{C}$. Membranes were then incubated with horseradish peroxidase-conjugated goat anti-rabbit IgG mAb (1:5,000; PV-6001; OriGene Technologies, Inc., Beijing, China) for $2 \mathrm{~h}$ at $37^{\circ} \mathrm{C}$. The blots were visualized using a chemiluminescence detection system (Invitrogen; Thermo Fisher Scientific, Inc.). All the experiments were performed in triplicate. Densitometric quantification of the immunoblot data was performed by Quantity-One software (version 2.0; Bio-Rad Laboratories, Inc.).

$N F-\kappa B$ overexpression. Renal cells $\left(1 \times 10^{5}\right)$ were isolated from experimental type $2 \mathrm{DM}$ rats before treatments as described previously (17) and were cultured in in RPMI 1640 medium supplemented with $10 \%$ heat-inactivated fetal bovine serum (FBS; both Thermo Fisher Scientific, Inc.) in 6-well plates for $12 \mathrm{~h}$ at $37^{\circ} \mathrm{C}$ until $85 \%$ confluence. The media was then removed from the culture plate, followed by three PBS washes. Renal cells were transfected with 100 pmol lentivirus-NF- $\kappa \mathrm{B}(\mathrm{pNF}-\kappa \mathrm{B})$ or lentivirus-vector (pControl; both Invitrogen; Thermo Fisher Scientific, Inc.) using Lipofectamine 2000 (Thermo Fisher Scientific, Inc.) according to the manufacturer's protocol. $\mathrm{NF}-\kappa \mathrm{B}$-overexpressed renal cells were used for further analysis following transfection for $48 \mathrm{~h}$ at $37^{\circ} \mathrm{C}$.

Immunohistochemical analysis. Renal tissues were obtained at week 8 and fixed in $10 \%$ formaldehyde for $2 \mathrm{~h}$ at $37^{\circ} \mathrm{C}$. Paraffin-embedded renal tissue sections (4- $\mu \mathrm{m}$-thick) were prepared and epitope retrieval was performed using water-bath heating for further analysis (18). Following dehydration in graded ethanol (100, 95 and $85 \%)$ and xylene, tissue sections were deparaffinized in xylene, rehydrated in descending ethanol series, followed by blocking of endogenous peroxidase activity in $3 \%$ hydrogen peroxide for $10 \mathrm{~min}$ at $37^{\circ} \mathrm{C}$. The paraffin sections were treated with hydrogen peroxide $(3 \%)$ for $15 \mathrm{~min}$ and subsequently were blocked with $5 \%$ bovine serum albumin (Sigma-Aldrich; Merck KGaA) for $30 \mathrm{~min}$ at $37^{\circ} \mathrm{C}$. The tissue sections were incubated with rabbit anti-mouse CD3 (1:1,000; cat. no. ab1669, Abcam) and CD19 (1:1,000; cat. no. ab25232, Abcam) at $4^{\circ} \mathrm{C}$ for $12 \mathrm{~h}$. Sections were then incubated with an Alexa 488-labeled goat anti-rabbit IgG antibody (cat. no. A-11034; 1:400; Molecular Probes; Thermo Fisher Scientific, Inc.) at $37^{\circ} \mathrm{C}$ for $2 \mathrm{~h}$. Stained sections were examined using an inverted laser scanning microscope (LSM 410; Zeiss AG, Oberkochen, Germany). The semi-quantification of immunoreactivity on each slide was evaluated using ImageJ software (version 1.02; National Institutes of Health, Bethesda, MD, USA).

Statistical analysis. Data are expressed as the mean \pm standard error of mean. All data were analyzed using SPSS 

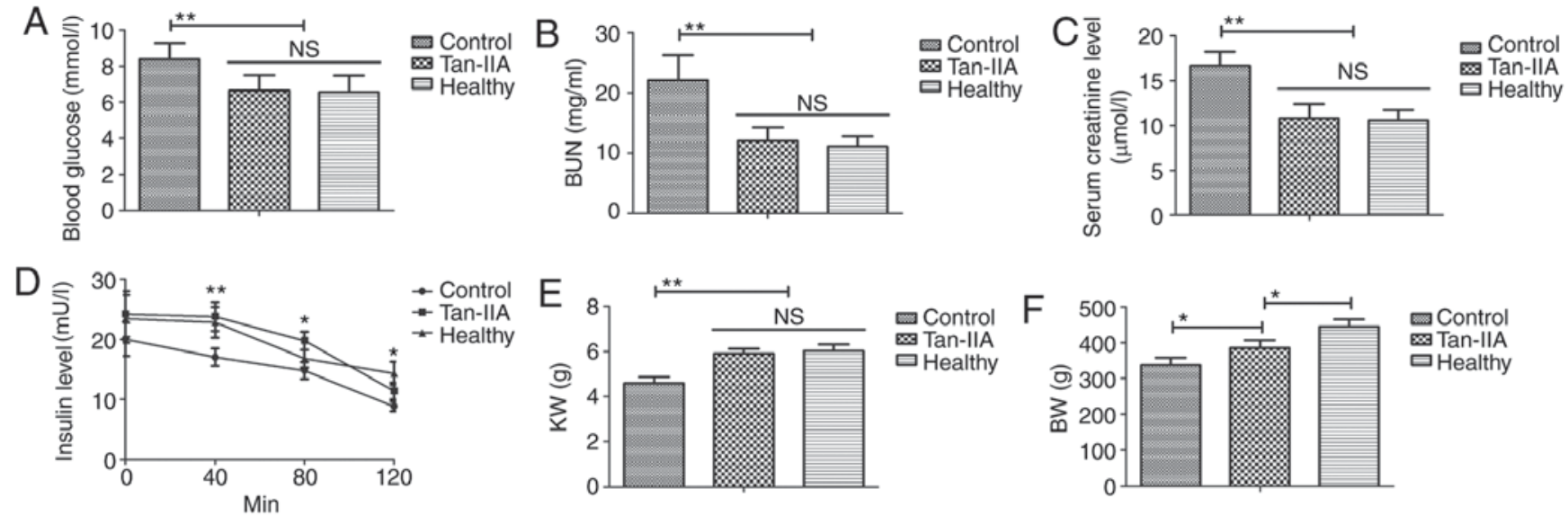

Figure 1. Tan-IIA treatment improves glucose metabolism and insulin resistance in type 2 DM rats. Effect of Tan-IIA treatment on (A) blood glucose, (B) serum BUN, (C) serum creatinine, (D) insulin resistance, (E) KW and (F) BW in type 2 DM rats. Data are expressed as the mean \pm standard error of the mean of three independent experiments. ${ }^{*} \mathrm{P}<0.05,{ }^{* *} \mathrm{P}<0.01$ vs. control group. NS, not significant; DM, diabetes mellitus; Tan-IIA, Tanshinone IIA; BUN, blood urea nitrogen; KW, kidney weight; BW, body weight.

version 16.0 (SPSS, Inc., Chicago, IL, USA). Groups were compared using one-way analysis of variance followed by a Student-Newman-Keuls test. $\mathrm{P}<0.05$ was considered to indicate a statistically significant difference.

\section{Results}

Tan-IIA treatment improves glucose metabolism and insulin resistance in type $2 \mathrm{DM}$ rats. As indicated in Fig. $1 \mathrm{~A}-\mathrm{C}$, Tan-IIA treatment significantly decreased blood glucose and serum levels of BUN and creatinine in type 2 DM rats compared with the control group $(\mathrm{P}<0.01)$. Tan-IIA treatment significantly improved insulin resistance for type $2 \mathrm{DM}$ rats compared with the control group (Fig. 1D; $\mathrm{P}<0.01$ at $40 \mathrm{~min}$, $\mathrm{P}<0.05$ at 80 and $120 \mathrm{~min})$. Notably, compared with the control, Tan-IIA treatment resulted in higher KW (Fig. 1E; P<0.01) and BW (Fig. 1F; $\mathrm{P}<0.05$ ). These results suggested that Tan-IIA treatment is beneficial for the improvement of glucose metabolism and insulin resistance in type 2 DM rats.

Tan-IIA treatment improves lipid metabolism for type 2 DM rats. As indicated in Fig. 2, it was observed that Tan-IIA treatment significantly reduced serum levels of TC, NEFAs, TG and total LDL-C in type 2 DM rats compared with the control group $(\mathrm{P}<0.01)$. These results suggested that Tan-IIA treatment is beneficial for improving lipid metabolism in type 2 DM rats.

Tan-IIA decreases inflammation in type 2 DM rats. As indicated in Fig. 3, it was demonstrated that Tan-IIA treatment significantly decreased levels of IL-8 $(\mathrm{P}<0.01)$, TNF- $\alpha$ $(\mathrm{P}<0.05), \mathrm{CRP}(\mathrm{P}<0.01)$ and IL-6 $(\mathrm{P}<0.05)$ compared with the control group. These results suggested that Tan-IIA treatment decreases inflammation in type $2 \mathrm{DM}$ rats.

Tan-IIA treatment reduces inflammation via the $N F-\kappa B$-induced AMPK signaling pathway. The possible mechanism of Tan-IIA in renal cells in type 2 DM was investigated. The results identified that Tan-IIA treatment significantly decreased expression levels of NF- $\kappa \mathrm{B}$ p65 $(\mathrm{P}<0.05$; Fig. 4A) and significantly elevated AMPK expression levels in renal cells $(\mathrm{P}<0.01$; Fig. 4A). Western blot analysis also indicated that Tan-IIA significantly elevated expression levels of IRS-1 $(\mathrm{P}<0.01$; Fig. 4B) and significantly decreased immunocyte precipitation in renal cells $(\mathrm{P}<0.01$; Fig. $4 \mathrm{C})$. Western blotting indicated that $\mathrm{NF}-\kappa \mathrm{B}$ overexpression $(\mathrm{pNF}-\kappa \mathrm{B})$ increased NF-kB p65 expression compared with the control and abolished Tan-IIA-increased AMPK levels in renal cells (both $\mathrm{P}<0.01$; Fig. 4D). NF- $\kappa \mathrm{B}$ overexpression also increased and blocked Tan-IIA-inhibited IL-8, TNF- $\alpha$, CRP and IL-6 in renal cells isolated from rats (all $\mathrm{P}<0.01$; Fig. $4 \mathrm{E})$. These results suggested that Tan-IIA treatment reduces inflammation via an $\mathrm{NF}-\kappa \mathrm{B}$-induced AMPK signaling pathway in renal cells of a type $2 \mathrm{DM}$ rat model.

\section{Discussion}

DM can lead to upregulation of inflammatory cytokines, which results in metabolic syndrome (19). A previous study described a novel mechanism for Tan-IIA in regulating vasorelaxation and may help to better understand the cardiovascular protective action of Tan-IIA (20). In the present study, the beneficial effects of Tan-IIA treatment for DM rats were investigated in vitro and in vivo. A dose of $10 \mathrm{mg} / \mathrm{kg}$ was used to evaluate the efficacy of Tan-IIA for DM, as described previously (21). The present findings indicated that Tan-IIA administration could decrease inflammatory cytokines, and alleviate glucose intolerance and insulin resistance in experimental rats. The current study also identified that Tan-IIA could improve insulin resistance in type $2 \mathrm{DM}$ mice via the $\mathrm{NF}-\kappa \mathrm{B}$-induced AMPK signaling pathway.

Inflammation is regarded as a central pathophysiological process in the development of type 2 DM (22). A previous study demonstrated that circulating IL-8 is associated with reduced insulin-like growth factor 1 and poor metabolism in adolescents with DM (23). A systematic review and meta-analysis indicated that serum TNF- $\alpha$ levels are upregulated in type $2 \mathrm{DM}$ patients, which is regarded as an elevated inflammatory burden in type 2 DM patients (24). In addition, CRP is a biomarker for patients with DM (25). Furthermore, a previous study revealed that IL-6 levels are 

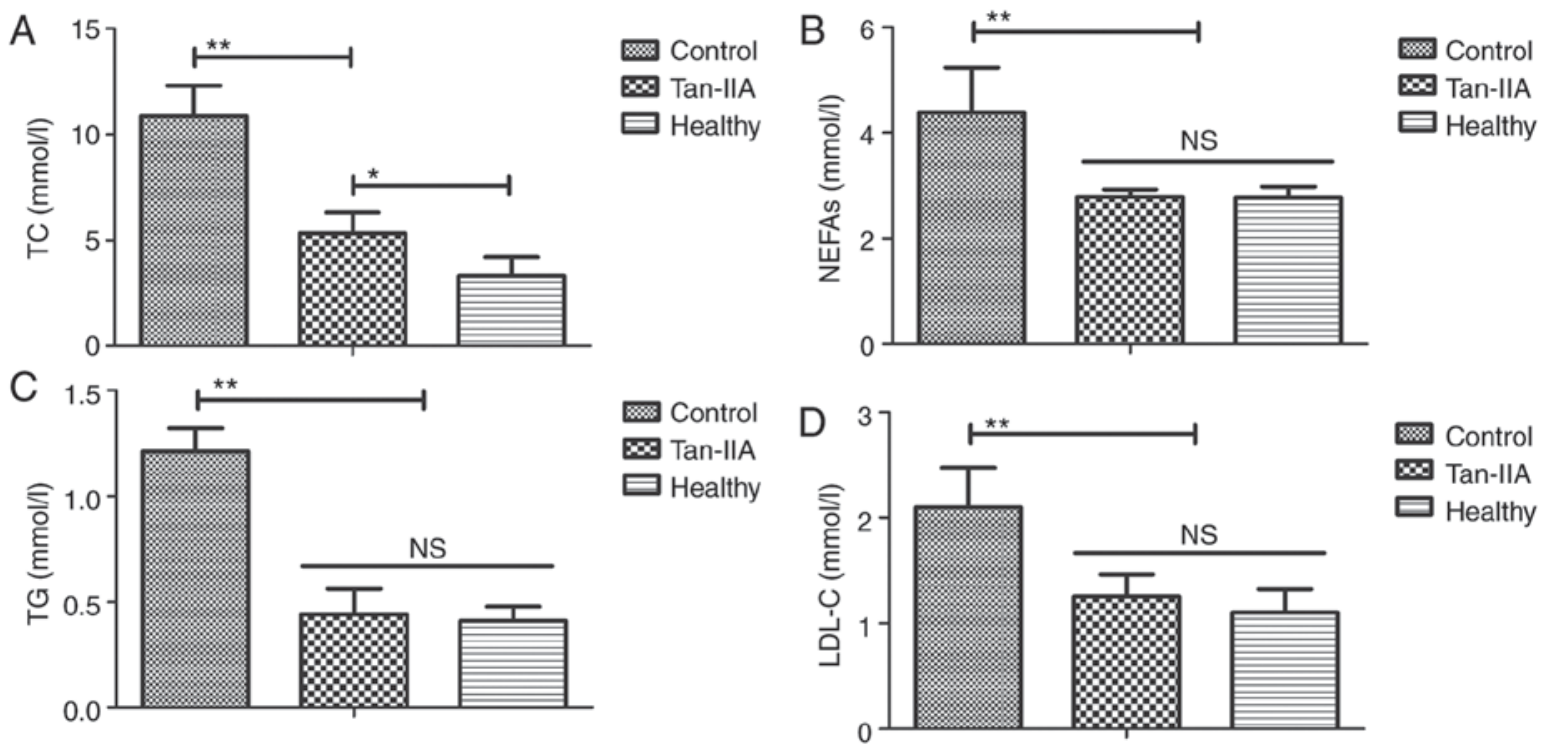

Figure 2. Tan-IIA treatment improves lipid metabolism in type 2 DM rats. Effect of Tan-IIA treatment on (A) TC, (B) NEFAs, (C) TG and (D) LDL-C in type 2 DM rats. Data are expressed as the mean \pm standard error of the mean of three independent experiments. ${ }^{* *} \mathrm{P}<0.01$ vs. control group. NS, not significant; DM, diabetes mellitus; Tan-IIA, Tanshinone IIA; TC, total cholesterol; NEFAs, non-esterified fatty acids; TG, total triglyceride; LDL-C, low density lipoprotein cholesterol.
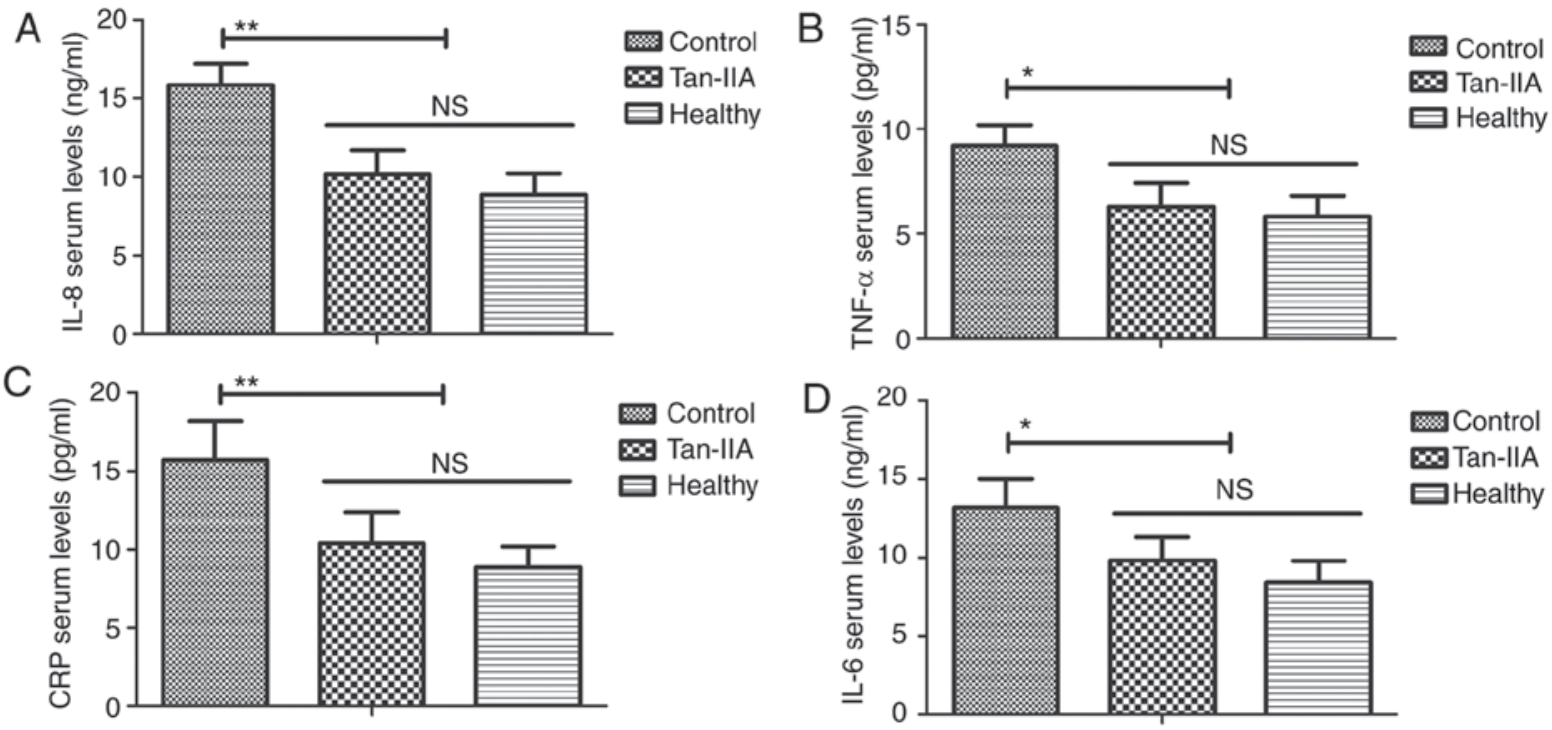

Figure 3. Tan-IIA decreases inflammation in type 2 DM rats. Effect of Tan-IIA treatment on (A) IL-8, (B) TNF- $\alpha$, (C) CRP and (D) IL-6 in type 2 DM rats. Data are expressed as the mean \pm standard error of the mean of three independent experiments. ${ }^{*} \mathrm{P}<0.05,{ }^{* * *} \mathrm{P}<0.01$ vs. control group. NS, not significant; DM, diabetes mellitus; Tan-IIA, Tanshinone IIA; IL, interleukin; TNF, tumor necrosis factor; CRP, C-reactive protein.

higher in patients with type 2 DM compared with healthy individuals (26). In the present study, it was observed that Tan-IIA treatment significantly decreased inflammatory factors TNF- $\alpha$, IL-6, CRP and Il-8 in a type 2 DM rat model. However, long-term experiments are required to verify these findings.

DM significantly affects blood glucose levels due to insufficient insulin concentration (27). It was observed in the current study that Tan-IIA treatment decreased blood glucose and reduced insulin resistance in DM rats. A previous report identified that serum levels of BUN and creatinine are higher in DM patients compared with healthy individuals (28). In the present study, it was demonstrated that Tan-IIA downregulated serum levels of BUN and creatinine, and increased KW and BW for type $2 \mathrm{DM}$ rats. Additionally, lipid metabolism disorder frequently occurs in DM patients (29). It was observed that Tan-IIA treatment significantly reduced TC, NEFAs, TG and LDL-C compared with the control group in type 2 DM rats.

NF- $\mathrm{KB}$ is involved in inflammation and insulin resistance in adipose cells in type $2 \mathrm{DM}$, and contributes to inhibition of inflammation and improves insulin resistance (30). In the present study, it was observed that NF- $\mathrm{kB}$ expression levels were increased in type $2 \mathrm{DM}$ rats and downregulated by Tan-IIA treatment. A previous report demonstrated that targeting of the IRS-1 pathway may regulate insulin resistance in type 2 DM rats (31). In the present study, Tan-IIA elevated expression 
A

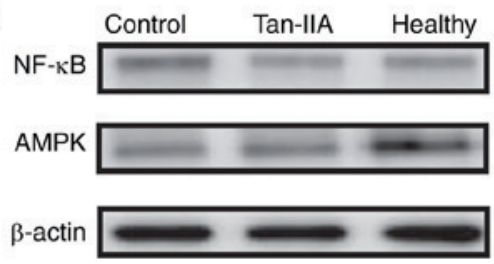

B

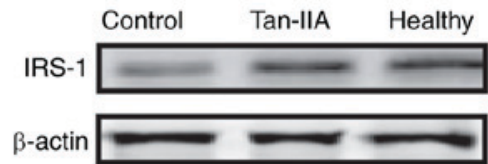

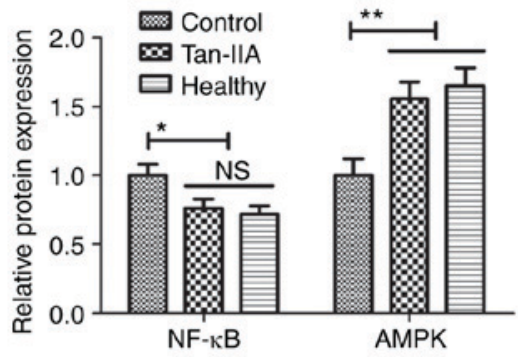

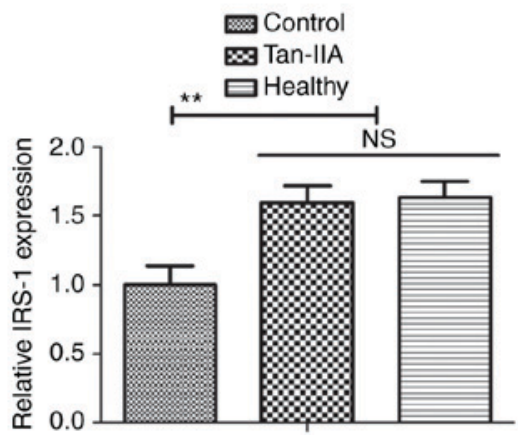

C
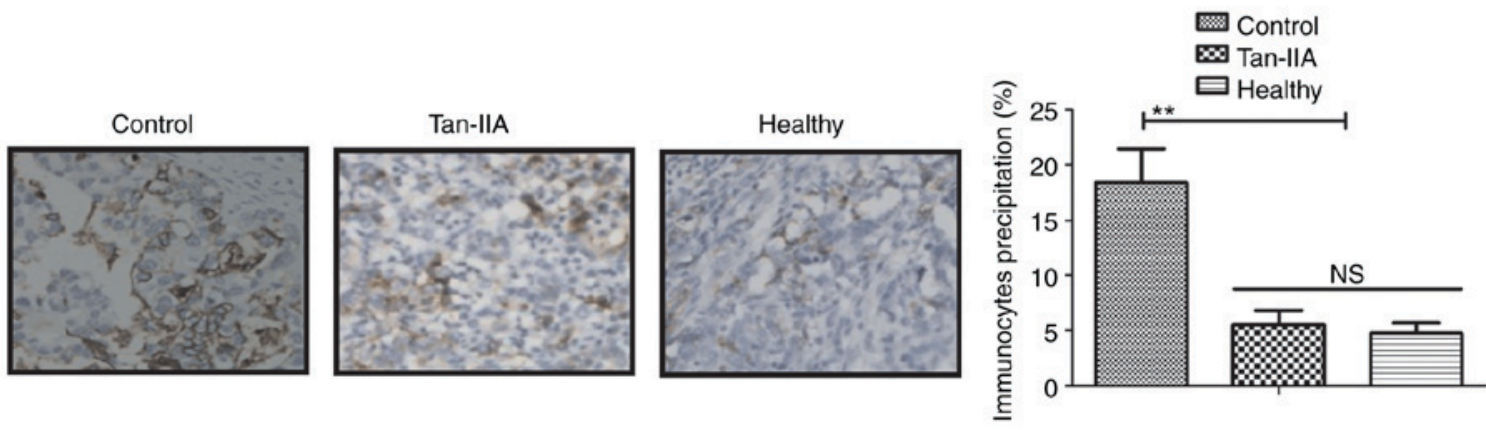

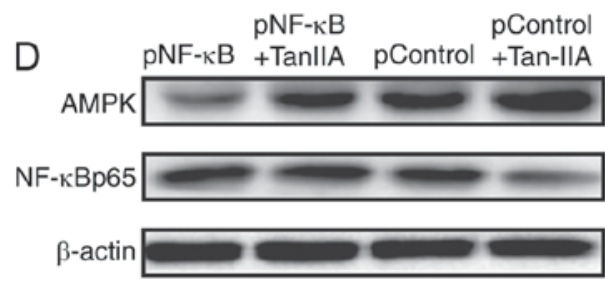

$\mathrm{E}$

pNF-кB

pControl

E pNF-kB +TanlIA pControl +Tan-IIA

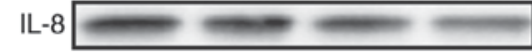

TNF- $\alpha \longrightarrow$ w w w

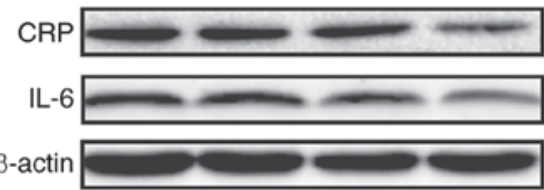

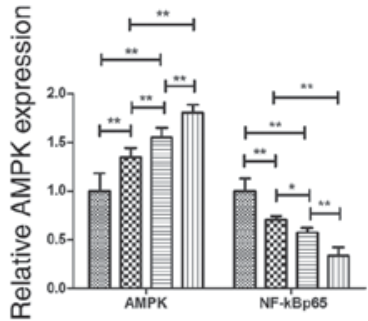

N.

$\circledast$ pNF-кB+TanllA

$\square$ pControl

西 pControl+Tan-IIA

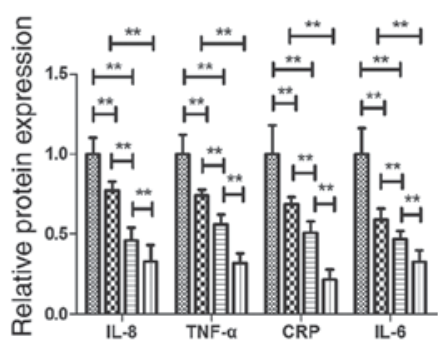

DNF-KB

$\otimes$ pNF-кB+TanlIA

口 pControl

四 pControl+Tan-IIA

Figure 4. Tan-IIA treatment improves type 2 DM via the NF-кB-induced AMPK signaling pathway. (A) Effect of Tan-IIA treatment on the expression level of NF-кB and levels of AMPK in renal cells. (B) Effect of Tan-IIA on the expression levels of IRS-1 in renal cells. (C) Effect of Tan-IIA treatment decreases

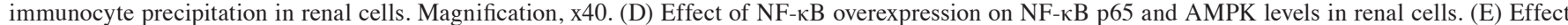
of NF- $\mathrm{kB}$ overexpression on IL-8, TNF- $\alpha$, CRP and IL- 6 expression in renal cells. Data are expressed as the mean \pm standard error of the mean of three independent experiments. "P $<0.05,{ }^{* *} \mathrm{P}<0.01$ vs. control group. NS, not significant; DM, diabetes mellitus; Tan-IIA, Tanshinone IIA; AMPK, 5' adenosine

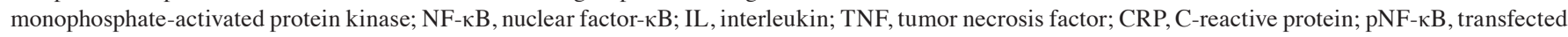
with NF-kB overexpression vector; pControl, transfected with control vector.

levels of IRS-1 in renal cells in type 2 DM rats. Upregulation of AMPK levels following Tan-IIA treatment was first reported in renal cells, and may further reduce production and activity of glucose-6-phosphatase in type 2 DM rats (32). However, a limitation of the present study was that only the number of immunocytes and expression of inflammatory cytokine 
levels were measured in renal cells. Further studies should be performed to analyze immunocytes and inflammatory cytokine expression in liver or adipose tissues in type $2 \mathrm{DM}$.

In conclusion, the present study indicates that Tan-IIA may have beneficial effects for treating type $2 \mathrm{DM}$ rats. The findings suggest that Tan-IIA treatment improves type $2 \mathrm{DM}$ via regulation of the NF- $\kappa \mathrm{B}$-induced AMPK signaling pathway. However, further studies are required to identify the efficacy of Tan-IIA in patients with type $2 \mathrm{DM}$.

\section{Acknowledgements}

Not applicable.

\section{Funding}

The present study was supported by Cadre Health Care Program of Sichuan Province as part of the following study, 'Study on the role of islet alfa cell function in the initiation and development of type 2 diabetes mellitus and the mechanisms of related drug intervention' (grant no. 2012-202, 30305030325).

\section{Availability of data and materials}

The analyzed data sets generated during the study are available from the corresponding author on reasonable request.

\section{Authors' contributions}

FYY performed the experiments. MZ designed the experiments. PX, DX, PC, MR, QS, JYS, JD and XLT prepared the investigations and analyzed data.

\section{Ethics approval and consent to participate}

The protocols were approved by the Ethics Committee of Sichuan Province People's Hospital \& Sichuan Academy of Medical Sciences (Chengdu, China).

\section{Patient consent for publication}

Not applicable.

\section{Competing interests}

The authors declare that they have no competing interests.

\section{References}

1. Bgeginski R, Ribeiro PAB, Mottola MF and Ramos JGL: Effects of weekly supervised exercise or physical activity counseling on fasting blood glucose in women diagnosed with gestational diabetes mellitus: A systematic review and meta-analysis of randomized trials. J Diabetes 9: 1023-1032, 2017.

2. Moosazadeh M, Asemi Z, Lankarani KB, Tabrizi R, Maharlouei N, Naghibzadeh-Tahami A, Yousefzadeh G, Sadeghi R, Khatibi SR, Afshari M, et al: Family history of diabetes and the risk of gestational diabetes mellitus in Iran: A systematic review and meta-analysis. Diabetes Metab Syndr 11 (Suppl 1): S99-S104, 2017.

3. Gyawali B, Ferrario A, van Teijlingen E and Kallestrup P: Challenges in diabetes mellitus type 2 management in Nepal: A literature review. Glob Health Action 9: 31704, 2016.
4. Mustafa SB, Mehmood Z, Akhter N, Kauser A, Hussain I, Rashid A, Akram M, Tahir IM, Munir N, Riaz M, et al: Review-medicinal plants and management of diabetes mellitus: A review. Pak J Pharm Sci 29 (Suppl 5): S1885-S1891, 2016.

5. Gomes JMG, Costa JA and Alfenas RCG: Metabolic endotoxemia and diabetes mellitus: A systematic review. Metabolism 68: 133-144, 2017.

6. Mizamtsidi M, Paschou SA, Grapsa J and Vryonidou A: Diabetic cardiomyopathy: A clinical entity or a cluster of molecular heart changes? Eur J Clin Invest 46: 947-953, 2016.

7. Shang Y, Zhang X, Chen L, Leng W, Lei X, Yang Q, Liang Z and Wang J: Assessment of left ventricular structural remodelling in patients with diabetic cardiomyopathy by cardiovascular magnetic resonance. J Diabetes Res 2016: 4786925, 2016.

8. Feng J, Li S and Chen H: Tanshinone IIA inhibits myocardial remodeling induced by pressure overload via suppressing oxidative stress and inflammation: Possible role of silent information regulator 1. Eur J Pharmacol 791: 632-639, 2016.

9. Shu M, Hu XR, Hung ZA, Huang DD and Zhang S: Effects of tanshinone IIA on fibrosis in a rat model of cirrhosis through heme oxygenase-1, inflammation, oxidative stress and apoptosis. Mol Med Rep 13: 3036-3042, 2016.

10. Lu Q, Zhang P, Zhang $X$ and Chen J: Experimental study of the anti-cancer mechanism of tanshinone IIA against human breast cancer. Int J Mol Med 24: 773-780, 2009.

11. Xu M, Cao FL, Zhang YF, Shan L, Jiang XL, An XJ, Xu W, Liu XZ and Wang XY: Tanshinone IIA therapeutically reduces LPS-induced acute lung injury by inhibiting inflammation and apoptosis in mice. Acta Pharmacol Sin 36: 179-187, 2015.

12. Wang DT, Huang RH, Cheng X, Zhang ZH, Yang YJ and Lin X: Tanshinone IIA attenuates renal fibrosis and inflammation via altering expression of TGF- $\beta /$ Smad and NF-kappaB signaling pathway in 5/6 nephrectomized rats. Int Immunopharmacol 26: 4-12, 2015 .

13. Han F, Sheng L, Jian Q and Weng Q: Therapeutic effects of Tanshinone IIA towards early renal damage in patients with type 2 diabetes. West Indian Med J, 2016.

14. Karabulut A, Akyer SP, Abban Mete G and Sahin B: Effects of menopause, diabetes mellitus and steroid use on type I mesh-induced tissue reaction in a rat model. Eur J Obstet Gynecol Reprod Biol 179: 27-31, 2014.

15. Galea R, Wells RG, Ross CK, Lockwood J, Moore K, Harvey JT and Isensee GH: A comparison of rat SPECT images obtained using $99^{\mathrm{m}} \mathrm{Tc}$ derived from ${ }^{99} \mathrm{Mo}$ produced by an electron accelerator with that from a reactor. Phys Med Biol 58: 2737-2750, 2013.

16. Arbillaga L, Vettorazzi A, Gil AG, van Delft JH, García-Jalón JA and López de Cerain A: Gene expression changes induced by ochratoxin A in renal and hepatic tissues of male F344 rat after oral repeated administration. Toxicol Appl Pharmacol 230: 197-207, 2008.

17. $\mathrm{Hu} \mathrm{H}, \mathrm{Xu} \mathrm{S}, \mathrm{Hu} \mathrm{S}, \mathrm{Gao} \mathrm{Y}$ and Shui H: Effect of $1,25(\mathrm{OH})_{2} \mathrm{D}_{3}$ on transdifferentiation of rat renal tubular epithelial cells induced by high glucose. Biomed Rep 5: 699-704, 2016.

18. Myers J: Antigen retrieval: A review of commonly used methods and devices. MLO Med Lab Obs 38: 10, 12-15; quiz 16-17, 2006.

19. Barami K, Lyon L and Conell C: Type 2 diabetes mellitus and glioblastoma multiforme-assessing risk and survival: Results of a large retrospective study and systematic review of the literature. World Neurosurg 106: 300-307, 2017.

20. Li YH, Xu Q, Xu WH, Guo XH, Zhang S and Chen YD: Mechanisms of protection against diabetes-induced impairment of endothelium-dependent vasorelaxation by Tanshinone IIA. Biochim Biophys Acta 1850: 813-823, 2015.

21. Jiang P, Li C, Xiang Z and Jiao B: Tanshinone IIA reduces the risk of Alzheimer's disease by inhibiting iNOS, MMP2 and NFkappaBp65 transcription and translation in the temporal lobes of rat models of Alzheimer's disease. Mol Med Rep 10: 689-694, 2014

22. Hamar P: Role of regulatory micro RNAs in type 2 diabetes mellitus-related inflammation. Nucleic Acid Ther 22: 289-294, 2012.

23. Van Sickle BJ, Simmons J, Hall R, Raines M, Ness K and Spagnoli A: Increased circulating IL-8 is associated with reduced IGF-1 and related to poor metabolic control in adolescents with type 1 diabetes mellitus. Cytokine 48: 290-294, 2009.

24. Chen YL, Qiao YC, Xu Y, Ling W, Pan YH, Huang YC, Geng LJ, Zhao HL and Zhang XX: Serum TNF- $\alpha$ concentrations in type 2 diabetes mellitus patients and diabetic nephropathy patients: A systematic review and meta-analysis. Immunol Lett 186: 52-58, 2017. 
25. Kawada T: C-reactive protein, depressive symptoms and incident diabetes mellitus with special emphasis on physical activity. J Psychosom Res 78: 407, 2015.

26. Darko SN, Yar DD, Owusu-Dabo E, Awuah AA Dapaah W, Addofoh N, Salifu SP, Awua-Boateng NY and Adomako-Boateng F: Variations in levels of IL-6 and TNF- $\alpha$ in type 2 diabetes mellitus between rural and urban Ashanti Region of Ghana. BMC Endocr Disord 15: 50, 2015.

27. Khosrozadeh M, Heydari N and Abootalebi M: The effect of Abelmoschus Esculentus on blood levels of glucose in diabetes mellitus. Iran J Med Sci 41: S63, 2016.

28. Javadi S, Asri-Rezaei S and Allahverdizadeh M: Interrelationship of beta-2 microglobulin, blood urea nitrogen and creatinine in streptozotocin-induced diabetes mellitus in rabbits. Vet Res Forum 5: 7-11, 2014.
29. van Olden C, Groen AK and Nieuwdorp M: Role of intestinal microbiome in lipid and glucose metabolism in diabetes mellitus. Clin Ther 37: 1172-1177, 2015.

30. Zhong L, Luo Y, Huang C and Liu L: Effect of NF-kB decoy on insulin resistance of adipocytes from patients with type 2 diabetes mellitus. Diabetes Metabol 37: 520-526, 2011.

31. Cai S, Sun W, Fan Y, Guo X, Xu G, Xu T, Hou Y, Zhao B, Feng X and Liu T: Effect of mulberry leaf (Folium Mori) on insulin resistance via IRS-1/PI3K/Glut-4 signalling pathway in type 2 diabetes mellitus rats. Pharm Biol 54: 2685-2691, 2016.

32. Yao L, Wan J,Li H, Ding J, Wang Y, Wang X and Li M: Resveratrol relieves gestational diabetes mellitus in mice through activating AMPK. Reprod Biol Endocrinol 13: 118, 2015. 